\title{
Effect of co-operative fuzzy c-means clustering on estimates of three parameters AVA inversion
}

\author{
RAJesh R NAIR* and SuREsh Ch KANDPAL \\ Department of Geology and Geophysics, Indian Institute of Technology, Kharagpur 721 302, India. \\ *e-mail: rajeshnair.iitkgp@gmail.com
}

\begin{abstract}
We determine the degree of variation of model fitness, to a true model based on amplitude variation with angle (AVA) methodology for a synthetic gas hydrate model, using co-operative fuzzy c-means clustering, constrained to a rock physics model. When a homogeneous starting model is used, with only traditional least squares optimization scheme for inversion, the variance of the parameters is found to be comparatively high. In this co-operative methodology, the output from the least squares inversion is fed as an input to the fuzzy scheme. Tests with co-operative inversion using fuzzy c-means with damped least squares technique and constraints derived from empirical relationship based on rock properties model show improved stability, model fitness and variance for all the three parameters in comparison with the standard inversion alone.
\end{abstract}

\section{Introduction}

The estimation of elastic parameters like P-wave velocity $\left(V_{p}\right)$, S-wave velocity $\left(V_{s}\right)$ and density $(\rho)$ across an interface can be found under optimal circumstances, using AVA (amplitude variation with angle) information (Aki and Richards 2002). The AVA is mainly governed by Zoeppritz equation (Zoeppritz 1919). The angle-dependent reflection coefficient at an interface separating two semiinfinite isotropic elastic media in concise matrix form is demonstrated by Aki and Richards (2002). Many approximations to Zoeppritz equation have been made by several authors (Wang 1999; Aki and Richards 2002). These approximations are focussed to give an understanding of how these amplitudes are related to various physical parameters. They are valid for small angle of incidence and low impedance contrast. To exploit the full information contained in the AVA data, we need to use the exact equations. Demirbag et al (1993) demonstrate that the exact densities are quite complicated to determine as the equation represents relative density and the convergence of $V_{s}$ (shear wave velocity) is also not so good with respect to $V_{p}$ (compressional velocity) for small offsets. So, we have these three parameters $V_{p}, V_{s}$ and $\rho$ and either one or two parameters (e.g., $V_{s}$ and $\rho$ ) always have large degree of variation in model fitness. Wang (1999) demonstrate new approximations to the Zoeppritz equations and their use in AVO analysis. The angles of incident, reflected and transmitted rays synchronous at the boundary are related according to Snell's law. The reflection coefficient at any given angle of incidence is completely determined by the P-wave, S-wave velocities and density of each medium. Therefore, the major task in AVA analysis is to restore relative amplitudes (AVA processing) in seismic prestack records before extraction and interpretation of the amplitude information. In exploration geophysics, we rarely deal with simple isolated interfaces. However, we must begin our understanding of offset-dependent reflectivity with the partitioning of energy at such an interface (Ostrander 1984; Castagna et al 1993).

We therefore test the present scheme of cooperative least squares and fuzzy c-means clustering on a synthetic hydrate model to investigate improvement in the estimation of density,

Keywords. AVA inversion; reflection coefficient; fuzzy logic; co-operative algorithm; cluster analysis. 
and improvement of the model fitness and stability compared to that for a three parameters AVA inversion. We approach a nonlinear multiparameter inversion technique with fuzzy clustering combined with a least squares inversion representing a co-operative methodology. This inversion scheme applies to cases for which there is well $\log$ information along with the seismic data, for instance in applications to quantify the gas hydrate and free gas accumulation.

\section{AVA method and fuzzy c-mean technique}

We perform a nonlinear AVA inversion for estimation of $V_{p}, V_{s}$ and $\rho$ and ' $\varepsilon$ ' (error function). The nonlinear problem can be stated as follows:

$$
R p p=f\left(\frac{\Delta V_{s}}{V_{s}}, \frac{\Delta V_{p}}{V_{p}}, \frac{\Delta \rho}{\rho}, \theta\right)+\varepsilon .
$$

The basic assumptions made are that the Earth has approximately horizontal layers at each common depth point, and that each layer is described by the three parameters $V_{p}, V_{s}$ and $\rho$ (Zoeppritz 1919; Aki and Richards 2002).

The parameter vector to be optimized comprises $n V_{s}$ values, followed by $n V_{p}$ values and $n \rho$ values where $n$ is the number of layers (we took $n=35$ equal to the total depth of layers and thus defined the pseudo layers in terms of constant thickness) such as:

$$
P=\left[V_{S 1} \ldots V_{S n}, V_{P 1} \ldots V_{P n}, \rho_{1} \ldots \rho_{n}\right] .
$$

From a given starting model $P_{\text {start }}$, the zero-offset $V_{s}, V_{p}$ and density reflectivity at an interface $i$ can be calculated as:

$$
\begin{aligned}
\frac{\Delta V_{S}}{V_{S}} & =\frac{V_{S}^{i}-V_{S}^{i-1}}{\left(V_{S}^{i}+V_{S}^{i-1}\right) / 2}, \\
\frac{\Delta V_{P}}{V_{P}} & =\frac{V_{P}^{i}-V_{P}^{i-1}}{\left(V_{P}^{i}+V_{P}^{i-1}\right) / 2} \\
\frac{\Delta \rho}{\rho} & =\frac{\rho^{i}-\rho^{i-1}}{\left(\rho^{i}+\rho^{i-1}\right) / 2}
\end{aligned}
$$

We then computed the reflection coefficient $R p p(\theta)$ for each angle and at each layer boundary. A synthetic offset seismic gather can be calculated by convolving the reflection coefficients $\operatorname{Rpp}(\theta)$ with predetermined wavelets. These synthetic data are compared with the observed data to form a misfit function. We then perturb each parameter of the solution vector in turn to form a new earth model, and generate new synthetic data, which are compared with the observation again. The process is repeated until the root mean square error (RMSE) function between the observed and the synthetic data is minimized. Constable et al (1990) show that by parametrizing the model in terms of its first or second derivatives with depth, the minimum norm solution yields the smoothest possible model. The above procedure can be described within an optimization framework: find a global minimum of a multivariable objective function. Once the global minimum is found, the corresponding model parameters are the resultant earth model. To reduce the non-uniqueness problem, the inversion algorithm is constrained by bounding the solution within a range of physical solutions. A simple illustration of this process is given in figure 1 .

We use an $L_{2}$-norm error function for the objective function expressed as:

$$
F_{3}=\sum_{j=1}^{m} \sum_{i=1}^{n}\left\|S_{\mathrm{obs}}^{i j}-S_{\mathrm{mod}}^{i j}\right\|^{2}
$$

where $S_{\text {obs }}^{i j}$ is the observed seismic amplitude at time index $i$ and angle index $j, S_{\text {mod }}^{i j}$ is the synthetic seismic amplitude at time index $i$ and angle index $j, n$ is the number of samples in a seismic trace, and $m$ is the number of angles.

To calculate the objective function, we need to model synthetic seismic responses. We use a convolution modelling technique, to generate synthetic data. The convolution technique, assumes planewave propagation across the boundaries of horizontally homogeneous layers, and takes no account of the effects of geometrical divergence, inelastic absorption, wavelet dispersion, transmission losses, mode conversions and multiple reflections. So the seismic data must be pre-processed to eliminate these effects and to restore plane wave amplitudes of primary P-wave reflections. Note that, one can use the reflectivity technique by Kennett et al (1988) and complex slowness to include the effect of reverberation, attenuation and dispersion. The seismic source wavelet is a Ricker wavelet generally used in seismic modelling (Hoversten et al 2006). Before inversion, seismic amplitudes are normalized to the amplitudes of the synthetic gather.

In AVA analysis, we use observations in term of receivers kept at the Earth's surface to reconstruct the spatial distribution of an underlying geophysical parameter: reflection coefficient. Using 


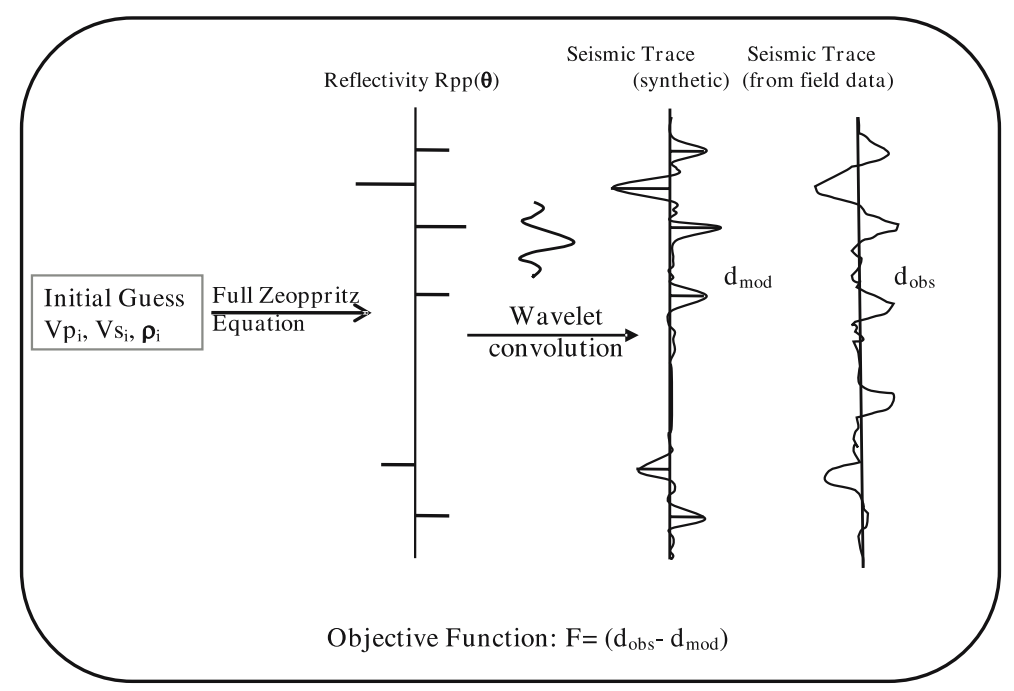

Figure 1. Workflow for nonlinear AVA inversion.

the simple least squares optimization for a multiparameter situation may not be the right choice, to deal with the non-uniqueness and instabilities. To be effective, the available datasets should be linked during the model generation process, for example, by a co-operative inversion. It is understood that the cluster analysis realises the information exchange between the individual models and data, but it cannot completely overcome the problems of inversion in several cases (Paasche and Tronicke 2007). We examine here, whether one can resolve the density parameter estimation using fuzzy c-means at least to a first order.

Fuzzy c-means (FCM) is a data clustering technique wherein each data point belongs to cluster to some degree that is specified by a membership grade. FCM play a roll of partitioning arbitrary $n$ vectors into $c$ fuzzy groups, also it finds a cluster center for each group such that it iteratively minimize objective function (Hoeppner et al 1999), that represents the distance from any given data point to a cluster center weighted by the data point's membership grade. Well-known fact about FCM indicates that FCM employs fuzzy partitioning such that a data point can belong to several groups with the degree of membership grades between zero and one. The optimum number of clusters $c$ is computed by minimizing the normalized classification entropy (NCE) (Lines et al 1988; Paasche et al 2006).

The normalized classification entropy introduced by Bezdek (1981) can be interpreted as a measure of cluster validity.

The classification entropy averages the quantity:

$$
h_{k}=-\sum_{i=1}^{c} u_{i j} \log _{a}\left(u_{i k}\right)
$$

and is defined by the function:

$$
H(U ; c)=-\sum_{k=1}^{q} \sum_{i=1}^{c} u_{i k} \log _{a}\left(u_{i k}\right) / q,
$$

where $a>1, U$ is the fuzzy membership matrix consisting of the fuzzy membership values corresponding to each cluster, $q$ is the number of observations within the dataset $X=\left\{x^{1}, x^{2}, \ldots, x^{q}\right\}$ and $c$ is the number of fuzzy classes. The value of $H$ ranges between zero and $\log _{a} c$. $H$ measures the ambiguity associated with a fuzzy partition. Naturally smaller the value of $H$, better is the cluster identification; zero being the ideal case. However, Bezdek (1981) argued that the endpoints of the range of $H$ (i.e., at $c=1, H=0, c=q$, $H=1$ ) do not give an accurate estimate of the disorganizations present within the clusters. So he introduced the concept of normalized classification entropy (NCE) defined as:

$$
\mathrm{NCE}=H(U ; c) /\left[1-\left(\frac{c}{q}\right)\right] .
$$

Fuzzy c-means (FCM) is based on minimizing the objective function (Bezdek 1981):

$$
J m=\sum_{i=1}^{N} \sum_{j=1}^{C} u_{i j}^{m}\left\|x_{i}-c_{j}\right\|^{2}, \quad 1 \leq m<\infty
$$

where $m$ is any real number greater than $1, u_{i j}$ is the degree of membership of $x_{i}$ in the cluster $j, x_{i}$ is the $i$ th component of the measured data (which is made by adding random noise, such that $S / N=4$, to the synthetically generated data ' $S_{0}$ ') 
using the equation $S_{0}=S_{0}+\left(\operatorname{rand}\left(\operatorname{size}\left(S_{0}\right)\right)\right) / 4$, $c_{j}$ is the d-dimension center of the cluster, and $\|*\|$ is any norm (whether L1, L2, etc.) expressing the similarity between any measured data and the center.

Fuzzy partitioning is carried out through an iterative optimization of the objective function, shown above, with the update of membership $u_{i j}$, the cluster centers $c_{j}$ (Jang et al 1997) by:

$$
\begin{aligned}
u_{i j} & =\frac{1}{\sum_{k=1}^{c}\left[\left\|x_{i}-c_{j}\right\| /\left\|x_{i}-c_{k}\right\|\right]^{2 /(m-1)}}, \\
c_{j} & =\frac{\sum_{i=1}^{N} u_{i j}^{m} \cdot x_{i}}{\sum_{i=1}^{N} u_{i j}^{m}} .
\end{aligned}
$$

This iteration, will stop when

$$
\max _{i j}\left\{\left|u_{i j}^{(k+1)}-u_{i j}^{(k)}\right|\right\}<\varepsilon,
$$

where $\varepsilon$ is a termination criterion, between zero and one, whereas $k$ are the iteration steps. This procedure converges to a local minimum or a saddle point of $J_{m}$.

The algorithm is composed of the following steps:

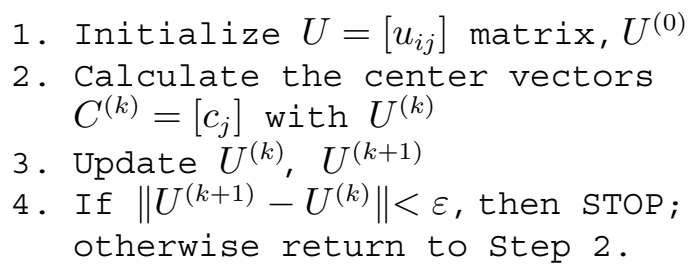

\section{FCM cluster analysis with damped least squares technique}

A union of conventional dataset inversion algorithm with FCM technique provides the sole theme of co-operative cluster inversion. To ensure information exchange between different geophysical datasets and models during the inversion process, at each iteration FCM cluster analysis is applied. First of all we define the $n$ different geophysical datasets $\left(V_{p}, V_{s}\right.$ and $\left.\rho\right)$, set the initial number of clusters to $C_{\min }=2$ and guess a reasonable number of clusters $C_{\max }$. Subsequently, equally discretized initial parameter models are generated using uniform squared grid cells for all datasets. Then FCM cluster analysis is performed on these $n$ individual models. Following this, membership information is used to calculate new model parameters for a cluster centre value weighted with the degree of membership of model cell to the $c$ clusters at each model cell. Now conventional regularized least squares single input data inversion is performed using new parameter models as input models. The RMSEs are computed from the corresponding forward calculations and the model updates for all the models. These updated models are then used in the following iteration, and we repeat this central inversion loop until the data misfits cannot be improved by further iterations. Here we define a tolerance value and give a condition that if the estimated value is greater than or equal to the tolerance value, then invoke the damped least squares technique, and update the initial model.

In the damped least squares technique, we minimize the residual that is defined as the sum of squares of the differences between the idealized model response and observed data. Input data vector is given as ' $d=G m$ ', where $G$ is the linear operator and $m$ is the unknown parameter vector velocity and density. We define $F=\left\{\|d-G m\|^{2}\right\}+\left(\alpha^{2}\|A m\|\right)^{2}$ where the first term represents the data misfit and the second term is the regularization term. The classical solution of the correction vector for an over-determined system using damped least squares method is given as $\delta p=\left(G^{T} G+\lambda I\right)^{-1} G^{T} z$, where $G$ is the linear operator and $G^{T}$ is the transpose of $G$ and $I$ is the identity matrix of the order of $G^{T} G$, while $\lambda$ is the damping factor which is used to prevent the solution from diverging to remove the ill conditionality of the matrix inversion problem.

As the optimum value of $c$ is usually not known a priori, we have to repeat the procedure using several reasonable values for $c$. The final model with the optimum $c$ is selected from these possible solutions by visually analyzing the calculated NCE and RMSE values. We seek the model with minimum complexity which concurrently minimizes the NCE values and the RMSEs. Figure 2 shows the flowchart showing the sequence of FCM clustering adapted for AVA Inversion. We have used least squares inverted output as the starting model for $V_{p}$ and $V_{s}$, while using the fuzzy clustering scheme.

\section{Synthetic data}

The input model for our synthetic example consists of five heterogeneous layer boundaries with acoustic impedance contrasts. The chosen model is typical for gas hydrates in a sedimentary setting, where the parameters tend to cluster within certain ideologies. The $V_{s}, V_{p}$ and density profiles correspond to published elastic properties of sediments containing hydrates (Dvorkin et al 1999). Aki and Richards equation (Florence et al 2004; Hoversten et al 2006) is used to compute the reflectivity 


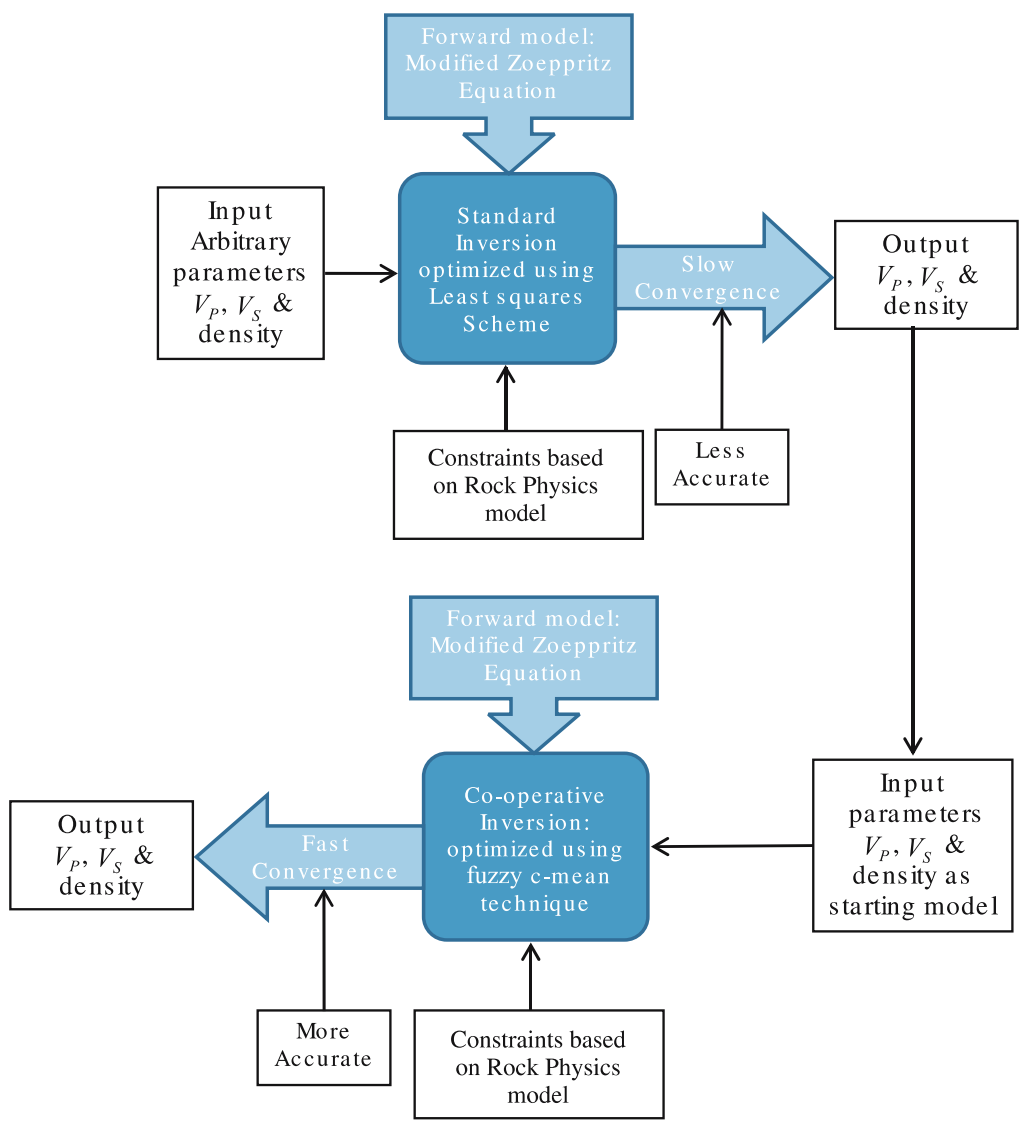

Figure 2. Flow chart showing the sequence of steps in the present scheme of fuzzy c-mean clustering adapted for AVA inversion.

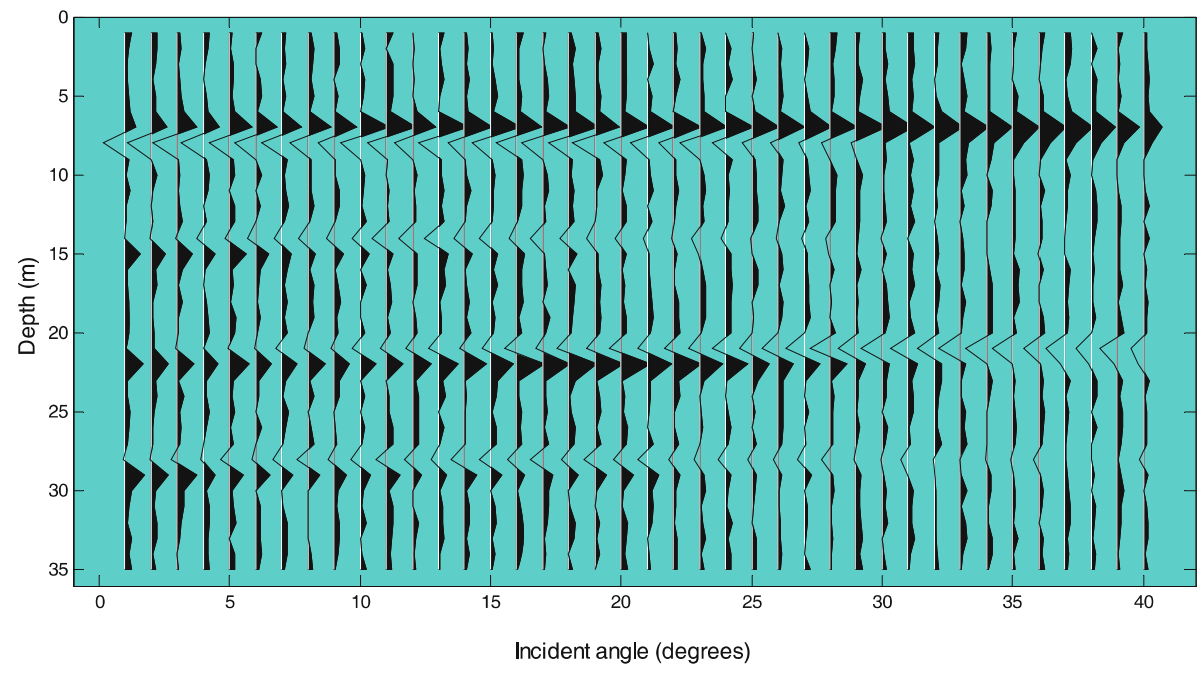

Figure 3. Forward modelled synthetic seismic data used for fuzzy c-means clustering.

profile for angles of $1-40^{\circ}$ corresponding to the angle range of the field data. Here we want to demonstrate the applicability and performance of the present inversion approach 'fuzzy c-means clustering', by linking the three parameters compressional velocity, shear velocity and density with a least squares AVA inversion. A synthetic offset seismic gather can be calculated by convolving the reflection coefficients $\operatorname{Rpp}(\theta)$ with predetermined Ricker wavelet. The synthetic data are added with a random noise component of $25 \%$ with respect to signal to make it a true data situation. Figure 3 

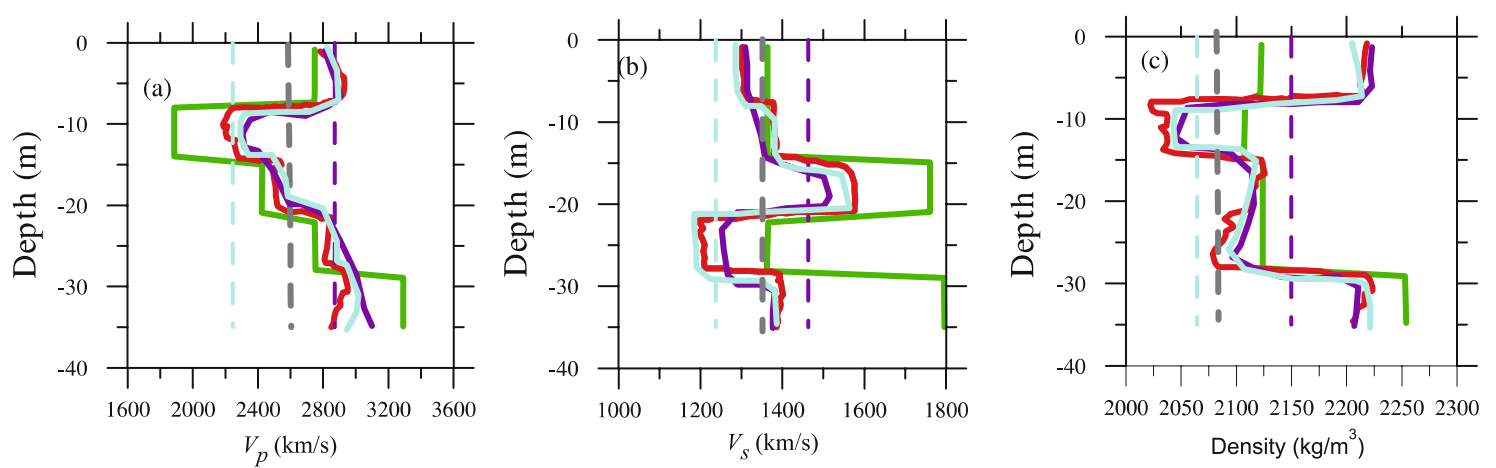

Figure 4. Results of standard inversion with optimization using least squares minimization scheme: Green line indicates true model, red line indicates inverted model and dashed line indicates the initial guess model. The initial guess models (three dotted lines), grey indicates the well log guided one and other two arbitrary guess model (sky blue and violet dotted lines) again used for fuzzy c-means inversion on either side of well log guided guess model. Least squares inversion with sky blue and violet dotted lines (initial guess model) are shown with same colours.

shows the forward modelled synthetic data, used as an input for the inversion scheme. These synthetic data are compared with the observed data to form a misfit function for the inversion scheme.

\section{Results of the standard inversion with least squares optimization}

The nonlinear inversion does not find the exact parameter values but the results are satisfactory. When adding noise to the data $(S / N=4)$, the unconstrained nonlinear inversion is not destabilized as the linear inversion. We conclude from the synthetic modelling that the nonlinear inversion is more robust than the linear inversion. The nonlinear inversion is little affected by the noise level in the data. Figure $4(\mathrm{a}-\mathrm{c})$ shows the initial guess model (three dotted lines), grey indicates the well log guided one and other two arbitrary guess model (sky blue and violet dotted lines) again used for fuzzy c-means inversion on either side of well log guided guess model. Least squares inversion with initial guess model (shown as sky blue and violet dotted lines) is shown with same colours. The misfit of $V_{p}, V_{s}$ and density and layer boundary fitness is higher based on least square inversion alone, with better fit obtained for the well log constrained initial guess in contrast with arbitrary lower and higher guess used in the inversion scheme.

\section{Results based on fuzzy c-means clustering approach}

We test the nonlinear inversion algorithm on a gas hydrate model, first by simple inversion based on least squares optimization scheme with homogenous layering as a starting model, and then by

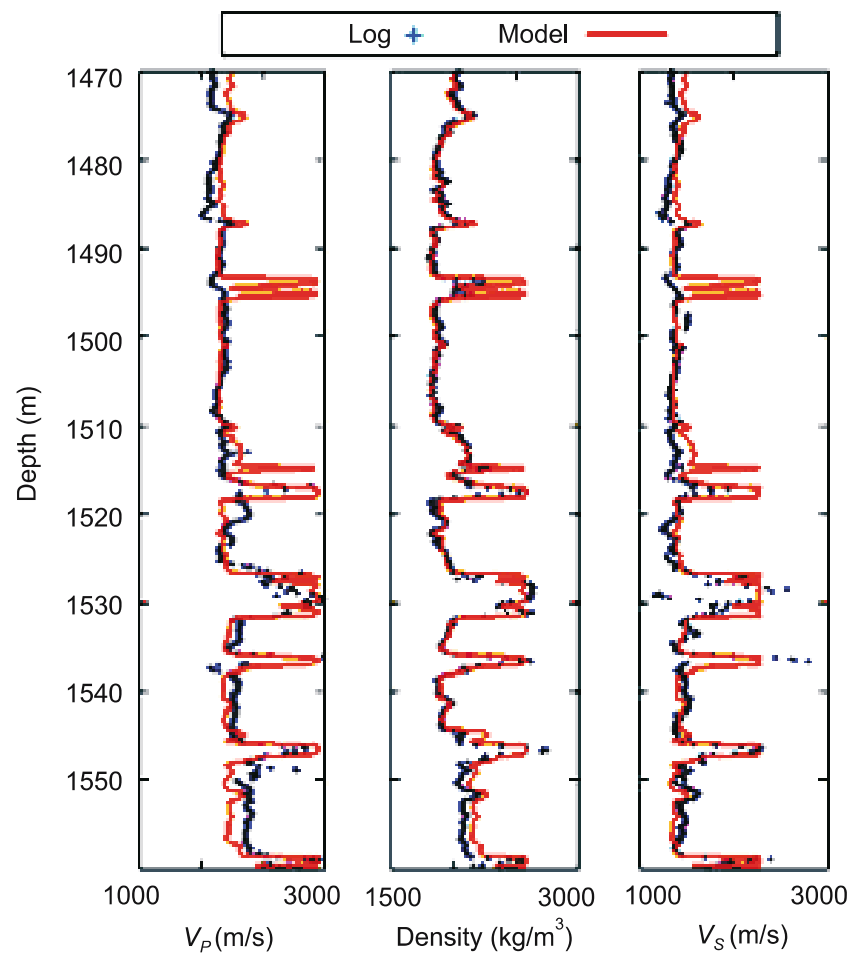

Figure 5. Computed $V_{p}, V_{s}$ and density data (red line) based on rock properties model (Hoversten et al 2006) in comparision with log (blue line) obtained corresponding data. The relationship between $V_{p}, V_{s}$ with that of density is used to provide a starting model for density alone in the present scheme.

means of fuzzy c-means applied using the cooperative fuzzy c-means clustering inversion.

The output from the least squares inversion is quite off from the true model. Therefore to avoid more searching, the empirical relationship derived from figure 5 (Hoversten et al 2006) is used as a constraint for the starting model for all three parameters. The parameters include critical porosity 0.38 , OIL API 28.5, brine salinity 0.07, 

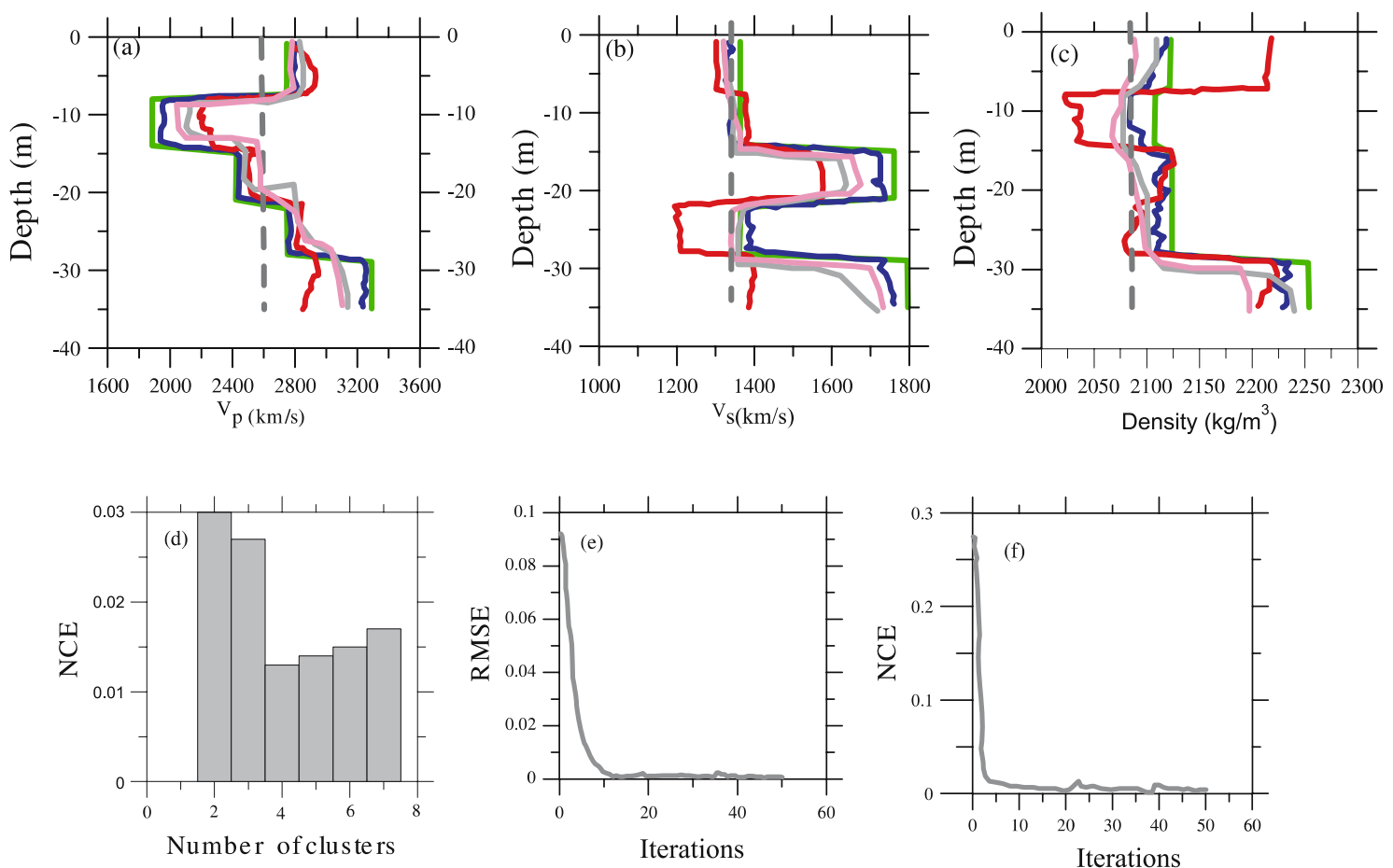

Figure 6. $\quad(\mathbf{a}-\mathbf{c})$ Fuzzy c-means inversion after least squares inverted results are given as input model. The blue line indicates inversion using fuzzy c-means optimization scheme, red standard inversion with least squares optimization scheme and green line indicates the true model. Fuzzy inversion fitness is shown as pink and grey colour curves of two other least square inputs for higher and lower values respectively with respect to figure $4(\mathrm{a}-\mathrm{c})$. (d) Indicates the minimum clusters for a lowest normalized classification entropy. (e) RMSE shows the parameters are obtained, when the error goes to minimum. (f) $\mathrm{NCE}$ values again indicates that results are obtained when the NCE values also goes to minimum.

gas gravity 0.59 , temperature $65^{\circ} \mathrm{C}$, grain shear modulus 22.5, grain Poissons ratio 0.34, grain density $2567 \mathrm{~kg} / \mathrm{m}^{3}$, number of contacts/grain 13.5 . Figure $6(\mathrm{a}-\mathrm{c})$ shows the fuzzy inversion shown as pink and grey colours of two other least square inputs for higher and lower values respectively with respect to figure $4(\mathrm{a}-\mathrm{c})$. The acceptable fitness percentage is not achieved in all cases (with three initial guess models) with least squares inversion alone. While fuzzy c-means gives best fitness for well $\log$ guided initial model and its least squares input, the fuzzy c-means fitness is not largely affected even if we use arbitrary guess model within some defined limits from the well log guided model. This shows the ability of the clustering algorithm in retrieving model parameters.

\section{Discussion}

AVA inversion with least squares alone shows that the $\mathrm{P}$-wave, S-wave and density does somewhat acceptable fitness, but not ideal for computations to estimate gas hydrate, free gas volume, etc., and shows lateral shift in layer boundaries. As said in the paper already, cluster analysis realizes the information exchange between the individual models/data, but it cannot overcome problems of the inversion itself. The cluster analysis will primarily make the individual layers more flat. Fuzzy cluster algorithm results in membership values/functions. For example, for our dataset, say the specific model point falls into cluster $x$ or $y$ or $z$, but not partially into several clusters as might be said by a fuzzy scheme. This process results in crisp models. In the case here, it is just the sorting of every model point into the cluster for which it has the highest membership to a particular cluster with no regard whether the membership is significantly or only a little bit higher than to another cluster.

When a co-operative inversion procedure is adopted, first with the starting model as a homogenous model, then we found that the co-operative fuzzy c-means gives a better fit to the model than the simple inversion with least squares, and further with least squares inverted output as a starting model, the convergence is better, with NCE and RMSE values going on to minimum. NCE reflects the degree of continuity and disorganization in the clustered data after classification. Since the minima of these functions, indicate the optimum number of classes where there exists a balance between continuity and structure, these 
Table 1. Collated table comparing the uncertainty in the inversion schemes for the three parameters.

\begin{tabular}{lccc}
\hline & \multicolumn{3}{c}{ Percentage error in } \\
\cline { 2 - 4 } Initial guess & $V_{p}$ & $V_{s}$ & $\rho$ \\
\hline Least squares $(l q)$ alone & & & \\
Lower value & 21.38 & 16.20 & 3.53 \\
Median value (well log & 14.45 & 14.71 & 3.83 \\
$\quad$ constraint) & & 19.28 & 3.63 \\
Higher value & 25.13 & & \\
Fuzzy c-means with least squares $(l q)$ as input & & 1.83 \\
Lower value derived lq & 5.57 & 3.31 & $\mathbf{0 . 6 8}$ \\
Well log value derived lq & $\mathbf{1 . 7 4}$ & $\mathbf{2 . 0 4}$ & 1.17 \\
Higher value derived lq & 7.00 & 4.62 & \\
\hline
\end{tabular}

results are useful as a guide to the choice of the number of classes. The results in figure 6 based on fuzzy c-means are close to the results of the true model. Faruk et al (2004) have reviewed the joint inversion of transient pressure and dc resistivity measurements acquired with in situ permanent sensors, shows that the co-operative inversion of $i n$ situ transient pressure and dc resistivity measurements reduce non-uniqueness in the estimation of resistivity and permeability governed by dynamic fluid flow phenomenon. Clearly, it makes sense to invert combinations of geophysical datasets in an attempt to gain additional subsurface information. The question then arises whether to invert the data jointly or sequentially (Treitel and Lines 2001). When one uses the joint inversion, in such a way that the output from one scheme is used as input to the other scheme, then the model will be better refined (Treitel and Lines 2001). In addition, joint inversions assume that physical properties co-vary, which is not always the case - arguing, in some instances, for the sequential inversion approach. We used here a similar scheme in inversion using fuzzy c-means with least squares output is given as input to the fuzzy clustering for inversion again. We agree that our scheme works much better when layer boundaries are well defined.

The velocity models resulting from the single inversion and co-operative inversions are shown in figures 4 and 6 , respectively. When analyzing the complete results, overall variability as well as the averaged velocity values of each layer, are indeed comparable to the corresponding characteristics of the input model used. This is shown here in figure 4, with poor model fit for single inversion and a better model fit for co-operative inversion as shown in figure 6 , when the starting model is the least squares inverted model. However, as the smoothness constraints influence the inversion process, the original clustered character of the velocity relation is not exactly reconstructed.
According to Occam's razor principle, we have chosen the four cluster solution that is the simplest model explaining both datasets - minimum RMSEs and satisfying our statistical criterion minimum NCE value. Since the whole analysis is done with signal-to-noise ratio 4 , it is found that results are little affected by the noise level in the data. We need not apply a misfit cut off (with multiple minima's absent), since the RMSE as shown in figure 6(e) indicates a single minimum, so our solutions are acceptable. Fuzzy c-means provided a best fit for the least squares input with well log guided guess model as shown in figure $4(\mathrm{a}-\mathrm{c})$ and retrieved model parameters figure $6(\mathrm{a}-\mathrm{c})$. Normalized classification entropy as shown in figure 6(f) is a kind of quality measure for the cluster analysis and again the simple analogy of RMSE as shown in figure $6(\mathrm{e})$, is yet another quality measure for the success of an inversion.

The collated table above is obtained after inversion results shown in figure $4(a-c)$ and figure $6(a-c)$ is used to compare the uncertainty in the least square inversion and fuzzy c-means. Least squares inversion alone shows that generally $V_{p}$ with an error of $14-25 \%, V_{s}$ shows $14-20 \%$ error and density $(\rho)$ of $3 \%$ only. Well log constrained least square inversion is better and most acceptable in comparison with the lower and higher guess values. Fuzzy c-means inversion shows the best acceptable percentage error $0.68-1.74 \%$ for $V_{p}, V_{s}$ and density $(\rho)$. Fuzzy c-means with least square inversion obtained better acceptable fitness for all the three initial guess values. A judicious combination of initial guess based on well log, with two cost functions and two optimization schemes (with output of one optimization fed as input to the second inversion scheme) with added fuzzy c-means capability of partitioning model parameters based on clustering with membership upgradation scheme resulted in deriving model parameters with acceptable level of fitness. This co-operative strategy emphasise 
the peculiarities of the inversion deriving better acceptable fitness.

\section{Conclusions}

We have reassessed the nature of model fitness and stability issue for AVA based scheme in inverting compressional, shear velocity and density. We used a co-operative fuzzy c-means cluster analysis combined with least squares scheme; reinforce the local character of the least squares technique by finding a local minimum of the error function and the well data allows constraining the solution to a region of the model space where the global minimum of the error function is located. Our results demonstrate a successful methodology to resolve the three parameters well. We conclude from the synthetic modelling that the co-operative fuzzy c-means nonlinear inversion is more robust than the conventional single optimization scheme algorithms for inversion. The simplicity and flexible approach of the scheme suggests the possibility for $2 \mathrm{D}$ and 3D problems for hydrocarbon exploration based on AVA. In our present case, we have shown co-operative inversion is better than standard inversion alone and when used in combination with standard inversion with some basic guess model from well log, it have given acceptable fitness. Although with a combined strategy, an acceptable fitness is obtained in the present case, our impression is that, its maximum benefit is not reached in all $1 \mathrm{D}$ problems, since it is a structurebased procedure and here structure exists only along one direction, in $2 \mathrm{D}$ or $3 \mathrm{D}$ problems this is more critical and there is more to do for a cluster analysis.

\section{Acknowledgements}

The authors sincerely thank Director, IIT Kharagpur for necessary facilities. Constructive suggestions by the reviewers helped to improve the paper. The support for this work was provided from the ISIRD project funded by IIT Kharagpur.

\section{References}

Aki K and Richards P G 2002 Quantitative Seismology; 2nd edn, University Science Books.
Bezdek J 1981 Pattern Recognition with Fuzzy Objective Function Algorithms; (New York and London: Plenum Press).

Castagna J P, Batzle M L and Kan T K 1993 Rock physics: The link between rock properties and AVO response; In: Offset-dependent reflectivity-theory and practice of AVO analysis (eds) Castagna J P and Backus M M, Soc. Expl. Geophys., pp 135-171.

Constable S C, Parker R L and Constable C G 1990 Occam inversion to generate smooth, two dimensional models from magnetotelluric data; Geophysics $\mathbf{5 5}$ 1613-1624.

Demirbag E, Coruh C and Costain J C 1993 Inversion of P-wave AVO; In: Offset-dependent reflectivity-theory and practice of $A V O$ analysis (eds) Castagna $\mathrm{J} \mathrm{P}$ and Backus M M, Soc. Expl. Geophys., pp 287-302.

Dvorkin J, Prasad M, Sakai A and Lavaie D 1999 Elasticity of marine sediments; Geophys. Res. Lett. 26 $1781-1784$.

Florence C, Rector J and Hoversten M 2004 Study of gas hydrates in the deep-sea Gulf of Mexico from seismic data; The Leading Edge 23 4356-4369.

Faruk et al 2004 Estimation of axisymmetric spatial distributions of permeability and porosity from pressuretransient data acquired with in situ permanent sensors; J. Petrol. Sci. Engg. 44(3-4) 231-267.

Hoeppener F, Klawonn F, Kruse R and Runkler T 1999 Fuzzy cluster analysis: Methods of classification, data analysis and image recognition; John Wiley and Sons, Inc.

Hoversten et al 2006 Direct reservoir parameter estimation using joint inversion of marine seismic AVA and CSEM data; Geophysics 71(3) C1-C13.

Jang J S R, Sun C T and Mizutani E 1997 Neuro-Fuzzy and Soft Computing; Prentice Hall.

Kennett B L N, Sambridge M S and Williamson P R 1988 Subspace methods for large inverse problems with multiple parameter classes; Geophys. J. Roy. Astron. Soc. 82 237-247.

Lines L R, Schulz A K and Treitel S 1988 Cooperative inversion of geophysical data; Geophysics $\mathbf{5 3} 8-20$.

Ostrander W J 1984 Plane-wave reflection coefficients for gas sands at nonnormal angles of incidence; Geophysics 49 1637-1649.

Paasche H and Tronicke J 2007 Cooperative inversion of 2D Geophysical data sets: A zonal approach based on Fuzzy c-means cluster analysis; Geophysics $\mathbf{7 2 ( 3 )}$ A35-A39.

Paasche H, Tronicke J, Holliger K, Green A G and Maurer H R 2006 Integration of diverse physicalproperty models: Subsurface zonation and petrophysical parameter estimation based on Fuzzy c-means cluster analyses; Geophysics 71(3) H33-H44.

Treitel S and Lines L 2001 Past, present and future of geophysical inversion - A new millennium analysis; Geophysics 66(1) 21-24.

Wang Y H 1999 Approximations to the Zoeppritz equations and their use in AVO analysis; Geophysics 64 1920-1927.

Zoeppritz R 1919 On the reflection and propagation of seismic waves; Exdbenwellen VIIIB, Gottinger Nchrichten I 66-84. 